\title{
Exploring the molecular world of turmeric: an alternative in the treatment of chronic inflammation
}

\begin{abstract}
Autoimmune and auto-inflammatory diseases affect over 50million Americans. Both of these disorders share common characteristics such as frequent episodes of inflammation followed by symptoms that may include rash, fever, joint pain, and joint swelling. These symptoms result when the body attacks its own tissue due to dysregulation of the innate immune system causing increased inflammation throughout the body. Over the past decade, numerous research articles have shows the benefit of complementary medicine and nutrition in the treatment of chronic inflammation. Despite evidenced based clinical research that supports the use of alternative medicine, many insurance companies and medical providers choose not to advocate for the use of these supplements. This paper reviewed the ongoing proposed benefits of Turmeric, a spice from the root Curcuma longa, a member of the ginger family as an anti-inflammatory agent. In-vitro and in-vivo research show various activities, such as anti-inflammatory, antiviral, antifungal, cytokines release, antioxidant, immunomodulatory, enhancing of the apoptotic process, and antiangiogenic properties of Turmeric. Challenges of treating and managing chronic inflammatory disease, and alternative treatment options were also highlighted.
\end{abstract}

Keywords: auto-inflammatory diseases, autoimmune diseases, chronic inflammation Curcumin, turmeric, antioxidant, antiviral, antifungal, cytokines, immunomodulatory, lupus erythematous, immune system, obesity, gout, atherosclerosis, osteoarthritis
Correspondence: Katrina Maree Trubilla, The Major General Hugh G Robinson Center for Neuropsychiatric Studies, US, Email author@robinsoncns.info

Received: February 07, 2017 | Published: February 20, 2017
Abbreviations: NSAIDS, no steroidal anti-inflammatory drugs; IBD, inflammatory bowel disease

\section{Introduction}

It is estimated that over 50million Americans are affected by chronic inflammation directly linked to an increase in autoimmune diseases, anti-inflammatory disorders; osteoarthritis; and the aging process. ${ }^{1}$ Extensive research has verified that continued oxidative stress and oxidative damage often results in chronic inflammation at the cellular level..$^{2-7}$ These cellular changes in turn mediate many chronic diseases that share clinically similar symptoms such as fevers, skin disorders, lymphadenopathy, joint pain; joint swelling, and musculoskeletal involvement. ${ }^{8}$ Inflammation is also linked to neurodegenerative diseases such as dementia and Alzheimer's diseases. ${ }^{7,9,10}$ Further, persistent inflammation is associated with chronic diseases including diabetes; cardiovascular disease; and obesity. $2,3,7,9,11,12$ Chronic inflammation often leads to chronic pain that can become debilitating interfering with quality of life. It is the purpose of this paper to review the ongoing proposed benefits of Turmeric; a spice from the root Curcuma longa, a member of the ginger family as an anti-inflammatory agent. In-vitro and in-vivo research show various activities, such as anti-inflammatory, antiviral, antifungal, cytokines release, antioxidant; immunomodulatory, enhancing of the apoptotic process, and antiangiogenic properties of Turmeric. This paper will also explore the challenges of treating and managing chronic inflammatory disease; and discuss alternative treatment options.

According to the American Autoimmune Related Diseases Association (AARDA), over 80 different autoimmune diseases have been identified. Autoimmune diseases develop when the immune system attacks the cells of the body confusing healthy cells as foreign cells. The most common diseases include rheumatoid arthritis, systemic lupus erythematous, celiac spur diseases, inflammatory bowel disease, psoriasis, Addison's disease, Hashimoto's disease, Graves' disease, and Sjogren's syndrome. ${ }^{1}$ The cause of autoimmune disease is unknown; however, possible triggers include bacteria, viruses, certain drugs, and chemical or environmental irritants. ${ }^{1}$ There is currently not a cure for autoimmune diseases, therefore, treatment involves symptom management especially during periods of exacerbations. ${ }^{1}$

Auto-inflammatory diseases are a relatively new classification of illnesses. The term "auto inflammatory" first appeared in the spring on 1999 to describe a host of clinical symptoms that were similar to autoimmune diseases, yet, different due to dysregulation of the innate immune system without auto reactive $\mathrm{T}$ lymphocytes and auto antibodies [8]. Auto inflammatory disorders are characterized by episodes of inflammation that result in fever, rash, or joint swelling, and can be potentially fatal due to vital organ involvement. ${ }^{2,8}$ The most common Auto inflammatory diseases include Familial Mediterranean Fever (FMF), Behcet's disease, Systemic Juvenile Idiopathic arthritis (Still's disease); Blau's disease; and Crohn's disease., ${ }^{2,8}$ As with autoimmune diseases, there is currently not a cure for autoinflammatory diseases. Treatment consists of managing symptoms and chronic inflammation. However, review of the literature shows that persistent; chronic inflammation is frequently related to secondary illnesses such type II diabetes, obesity, gout, atherosclerosis, osteoarthritis, and osteoporosis. Recent studies show that other pathophysiological processes not related to autoimmune or Auto inflammatory diseases respond with a wide spectrum of inflammation (aging; cancer, metabolic disorders, dementia, Alzheimer's, cardiovascular disease). ${ }^{2,8}$ Treatment for inflammatory disorders 
is complex. Research suggests that traditional pharmacological treatments have significant side effects and may result in additional health conditions after long-term use. ${ }^{7,11}$ A retrospective review of 3.2 million patients using two commonly over the counter nonselective no steroidal anti-inflammatory drugs (NSAIDS), naproxen, or ibuprofen with or without the use of aspirin showed a statistically significant increase in reported gastrointestinal (GI) toxicities defined as perforations; ulcers, and bleeds. ${ }^{7,11}$ Therefore, it is critical as health care providers to explore safe, alternative and complimentary treatment options to improve the long-term care of patients diagnosed with chronic inflammation.

\section{Discussion}

Diet and nutrition can have significant impact on the inflammatory process in the human body. ${ }^{4}$ Turmeric, a golden spice is obtained from the rhizome of the plant Curcuma longa and traditionally used to provide color and taste in food since ancient times. ${ }^{15}$ Turmeric is composed of three curcuminoids (curcumin, desmethoxycurcumin, bisdemethoxycurcumin) Although it was long believed that Turmeric was responsible for the beneficial health effects, research today has narrowed its focus to the component of Curcumin, as being primarily responsible. In fact, it is this agent that has been shown to regulate numerous transcription factors, cytokines, protein kinases, adhesion molecules, redox status and enzymes that have been linked to inflammation. ${ }^{9}$ several volatile oils, sugars, proteins, and resins. ${ }^{7}$ Extensive research shows that Curcuma longa acts as a multi-targeted agent that modulates multiple cell signaling pathways responsible for cellular inflammation. . $^{6,72,15}$

Curcumin, the major constituent of Curcuma longa, and a dietbased yellow color agent found in Turmeric has many scientific and health benefits. Curcumin is a Polyphenol with a lipophilic structure that is insoluble in water and stable in the acidic environment of the stomach. ${ }^{7,9,3,12,16}$ It is a highly Pleotropic molecule that frequently interact $\mathrm{s}$ with other molecular particles in the inflammatory process and has-strong anti-oxidative properties., ${ }^{3,9,10,14,18}$ The antiinflammatory mechanism of Curcumin is a molecular response to the down-regulation of enzymatic activity of cyclooxygenase-2 (COX2 ), lipoxygenase, and inducible nitric oxide synthase (iNOS). ${ }^{3,4,14}$ It also is modulated by other cell signaling activity involving cytokines, apoptotic proteins, NF-kB, 5-LOX, STAT3, reactive protein, prostaglandin E (2), adhesion molecules, creatinine; and a host of other critical molecules and enzymes. . $^{3,4,14}$

Since the first publication on the biological benefits of Curcumin in The Lancet of 1937, a further 9000 articles have been published describing the therapeutic benefits of turmeric as a nutraceutical. $3,9,16$ In particular, over 100 different clinical trials have been published showing the anti-inflammatory activity of Curcumin molecules.

The uniqueness of Curcumin makes it particularly effective in the treatment of organ and tissue disorders. It is used to help prevent and treat organ and tissue disorders associated with inflammation and oxidative stress. ${ }^{3,17}$ Its ability to regulate inflammatory and pro-inflammatory pathways helps alleviate oxidative stress and inflammation in chronic illnesses. ${ }^{3,7,12,14,15}$ Further, as an oral bioavailability blocker of TNF, it is an effective treatment for Inflammatory Bowel Disease (IBD), has antimicrobial properties ${ }^{19}$ and effectively inhibits carcinogenesis in various types of cancers inclusive of colorectal, pancreatic, gastric, prostate, hepatic, breast, oral, and leukemia, ${ }^{3,9,10,12}$ particularly, prostate cancer. ${ }^{19}$
There are also many other benefits to using Cur cumin. For example, the chemicals found in Tumeric are shown to be safe, easily tolerated and effective in the treatment of chronic diseases in humans..$^{5,12-14,16}$ Furthermore, the low cost and ease of availability also makes Turmeric an appealing alternative treatment option for chronic inflammation. ${ }^{13,14}$

Although Turmeric has been found to provide significant health benefits at a cost conducive to many, its use should be exercised with caution as there are many concerns not yet researched nor clarified. Because Curcumin has the unique molecular ability to prevent inflammation by acting on multiple sites, its synergistic effects with other drugs and/or chemicals remain largely unknown. Hence, current studies do not provide critical information on the safety of using Turmeric with other medications ${ }^{5}$ and any existing contraindications of such are new and unfolding. ${ }^{20}$ This area of study, therefore, requires further exploration. Further, to achieve therapeutic levels, Turmeric supplements are required in large dosages ${ }^{7,16,17}$ which is difficult to implement given that the interactions it creates are uncertain. In addition, there is a dearth of research to assess the long term effects of Turmeric. For example, ongoing reports of gastrointestinal disorders related to long-term use of pharmaceutical or over the counter NSAIDS, compel continual research into safer treatment options for patients. Finally, while most research to date includes rats and mice, longitudinal studies are needed to determine any possible long-term effects of high dosages of Turmeric in humans. Ongoing studies that examine dose-dependent relationships and limiting factors to effectiveness are still required. ${ }^{17}$

\section{Limitations}

Although numerous clinical studies have demonstrated the effectiveness and safety of using Turmeric and/or Curcumin as a nutraceutical in the prevention and treatment of chronic inflammation and many other disease processes; this review is not without its limitations. For example, many of the studies included in this article were reviews and absent of core primary research, ${ }^{10,13,15}$ omitted a methodology, ${ }^{6}$ and often had no clear definitive inclusion criteria; ${ }^{19,20}$ all of which infringes on the generalizability and validity of the findings. Further, this review used no systematic approach or inclusion/exclusion criteria to include and/or exclude the articles it did. Studies were determined and included primarily based on their relevance to the topic under discussion which ultimately resulted in a snowball effect to include any particular and relevant study. ${ }^{21}$

\section{Conclusion}

Nutritional interventions that use foods with anti-inflammatory chemical properties have proven to be a valuable treatment option in both the prevention and treatment of multiple chronic diseases, particularly those of an inflammatory nature. A review of the literature shows that Turmeric has the potential to be a safe, effective, and an affordable alternative or supplement in the treatment of chronic inflammation. Early cell culture and animal research, indicates that Curcumin is effective as a therapeutic agent for IBD, pancreatitis, lupus nephritis, osteoarthritis, and certain types of cancers. While research on the benefits of Turmeric, and its component of Curcumin, in particular, have multiplied substantially in recent years, there is still much research needed on determining the long term effects and potential interactions Curcumin imposes on humans. Something that perhaps, insurance companies await before making their decision to cover such a substance. 


\section{Acknowledgements}

Katrina Maree Trubilla, MD Student, USAT, Montserrat.

Lisa Adams, PhD, MD Student \& Faculty, USAT, Montserrat.

Tony Brown, MD, Faculty of Medicine, USAT, Montserrat.

\section{Conflict of interest}

The author declares no conflict of interest.

\section{References}

1. Schulze-Tanzil G, Mobasheri A, Sendzik J, et al. Effects of curcumin (difeuloymethane) on nuclear factor kappaB signaling in interleukin-1 beta-stimulated chondrocytes. Ann N Y Acad Sci. 2004;1030:578-586.

2. Ciccarelli F, De Martinis M, Ginaldi L. An update on Auto inflammatory diseases. Curr Med Chem. 2013;21(3):261-269.

3. Jurenka J. Anti-inflammatory properties of curcumin, a major constituent of Curcuma long: a review of preclinical and clinical research. Altern Med Rev. 2009;14(2):141-153.

4. Kunnumakkara AB, Bordoloi D, Padmavathi G, et al. Curcumin, the golden nutraceutical: multi-targeting for multiple chronic diseases. $\mathrm{Br} \mathrm{J}$ Pharmacol. 2017;174(11):1325-1348.

5. Shehzad A, Rehman G, Lee YS. Curcumin in inflammatory diseases. Biofactors. 2013;39(1):69-77.

6. Sun D, Zhuang X, Xiang X, et al. A novel nanoparticle drug delivery system: The anti-inflammatory activity of curcumin is enhances when encapsulated in exosomes. Mol Ther. 2010;18(9):1606-1614.

7. Thomas J, Straus WL, Bloom BS. Over-the-counter nonsteroidal anti-inflammatory drugs and risk of gastrointestinal symptoms. Am J Gastroenterol. 2002;97(9):2215-2219.

8. Sahebkar A, Saboni N, Pirro M, et al. Curcumin: An effective adjunct in patients with statin-associated muscle symptoms. J Cachexia Sarcopenia Muscle. 2017;8(1):19-24.

9. Aggarwal BB, Harikumar KB. Potential therapeutic effects of curcumin, the anti-inflammatory agent, against neurodegenerative, cardiovascular pulmonary, metabolic, autoimmune and neoplastic diseases. Int $J$ Biochem Cell Biol. 2009;41(1):40-59.
10. Gardener SL, Rainey-Smith SR, Martins RN. Diet and inflammation in Alzheimer's disease and related chronic diseases: A review. Journal J Alzheimers Dis. 2016;50(2):301-334.

11. Biskupiak JE, Brixner DI, Howard K, et al. Gastrointestinal complications of the over-the counter nonsteroidal anti-inflammatory drugs. J Pain Palliat Care Pharmacother. 2006;20(3):7-14.

12. Roddic J. Autoimmune Disease. Healthline. 2015.

13. Gupta SC, Kismali G, Aggarwal BB. Curcumin, a component of turmeric: from farm to pharmacy. Biofactors. 2013;39(1):2-13.

14. He Y, Yue Y, Zheng $\mathrm{X}$, et al. Curcumin, inflammation, and chronic diseases: How are they linked? Molecules. 2015;20(1):9183-9213.

15. Henrotin Y, Clutterbuck AL, Allaway D, et al. Biological actions of curcumin on articular chondrocytes. Osteoarthritis Cartilage. 2010;18(2):14-19.

16. National Institute of Health National Institutes of Arthritis and Musculoskeletal and Skin Diseases. Understanding Auto inflammatory Diseases. 2016: 1-4

17. Ghosh SS, Gehr TW, Ghosh S. Curcumin and chronic kidney disease (CKD):Major mode of action through stimulating endogenous intestinal alkaline phosphatase. Molecules. 2014;19(12):20139-20156.

18. Gupta SC, Sung B, Kim JH, et al. Multitargeting bytumeric, the golden spice: From kitchen to clinic. Mol Nutr Food Res. 2013;57(9):1510 1528.

19. Adahoun, MA Al-Akhras, MAH, et al. Enhanced anti-cancer and Antimicrobial activities of curcumin nanoparticles. Nanomedicine \& Biotechnology. 2017;45(1):98-107.

20. De Paz-Campos MA, Ortiz MI, Chávez Piña AE, et al. Synergistic effect of the interaction between curcumin and diclofenac on the formalin test in rats. Phytomedicine. 2014;21(12):1543-1548.

21. Gopalakrishna RN, Bannimath G, Huded SP. Herb-drug interaction: Effect of Poly-Herbal Formulation on Glibenclamide Therapy in Patients with Type-2 Diabetes Mellitus. Pharmaceutical Methods. 2017;8(1):200-208. 